\title{
Radiation therapy for primary tumor of de novo stage IV breast cancer
}

\author{
Michio Yoshimura \\ Department of Radiation Oncology and Image-applied Therapy, Kyoto University, Graduate School of Medicine, Kyoto, Japan \\ Correspondence to: Michio Yoshimura, MD, PhD. Department of Radiation Oncology and Image-applied Therapy, Kyoto University, Graduate School \\ of Medicine, 54 Kawahara-cho, Shogoin, Sakyo-ku, Kyoto, 606-8507, Japan. Email: myossy@kuhp.kyoto-u.ac.jp.
}

\begin{abstract}
Despite recent advances in multimodality treatments such as endocrine therapy, chemotherapy, molecularly targeted therapy, and radiation therapy, it is still very difficult to cure de novo stage IV breast cancer patients completely. The traditional role of radiation therapy for these patients has been a palliative treatment strategy that aims to control tumor progression and suppress tumor related symptoms. Recently, several non-randomized retrospective studies on de novo stage IV breast cancer have revealed that locoregional radiation therapy (LRRT) might confer a survival benefit. However, there is no high level evidence to support the impact of LRRT on survival among patients with de novo metastatic disease so far. This article aimed to summarize the literature and to discuss whether treating the primary lesion with radiation therapy could improve clinical survival outcomes among de novo stage IV breast cancer patients. The issue of patient selection will be discussed because not all de novo stage IV breast cancer patients could benefit from LRRT. This article also explores the clinical evidence regarding LRRT for de novo metastatic disease across various cancers such as prostate, uterine cervical, non-small-cell lung, and head and neck cancers. Many retrospective trials have shown the impact of locoregional treatment (LRT) on survival in de novo metastatic breast cancer. However, since the backgrounds of patients treated with LRRT are quite different from those of patients who did not receive LRRT and the treatment consists of surgery and/or radiation therapy, the role of radiation therapy alone remains unclear. Several reports investigated prognostic factors to detect the benefits of LRRT, which still remains conflicting and no consensus exists. However, selected patients with de novo metastatic disease with better performance status, low tumor burden, and estrogen receptor positivity should be considered for the addition of radiation therapy delivered to the primary site. To explore proper decision-making regarding LRRT, further prospective randomized trials are eagerly awaited.
\end{abstract}

Keywords: Breast cancer; radiation therapy; radiotherapy; de novo stage IV

Submitted Jan 27, 2020. Accepted for publication Feb 14, 2020.

doi: $10.21037 /$ tcr.2020.02.54

View this article at: http://dx.doi.org/10.21037/tcr.2020.02.54

\section{Introduction}

Breast cancer is the most common malignancy among women worldwide. About $3-10 \%$ of breast cancer patients have metastatic disease at the initial diagnosis, which is referred to as de novo stage IV breast cancer $(1,2)$. Recent advances in multimodality treatment strategies for breast cancer have improved the overall survival (OS) time of de novo stage IV breast cancer patients. However, the local control of the primary tumor is sometimes difficult and local recurrence leads to the loss of QOL (quality of life). Moreover, locoregional treatment (LRT) of the primary site in stage IV breast cancer patients may not only suppress local failure, but also reduce seeding from the primary site. Therefore, it is very important to suppress local failure of the primary lesion. According to the NCCN (National Comprehensive Cancer Network) guideline, the role and timing of surgical removal of the primary tumor among 
patients with de novo stage IV disease is the subject of ongoing investigations and must be individualized. Local breast surgery and/or radiation therapy are reasonable for selected patients who respond to initial systemic therapy. Retrospective trials have suggested a potential survival benefit from complete surgical resection of the primary tumor in selected patients with de novo stage IV breast cancer. A prospective randomized trial (MF07-01) (3) conducted by a Turkish group showed that the median survival time was longer in the surgical resection group compared with the no surgical resection group (46 vs. 37 months, $\mathrm{P}=0.005)$. However, two randomized trials published by an Indian group (4) and an Austrian group (5) could not demonstrate any survival benefit for surgical resection of the primary tumor among breast cancer patients with de novo stage IV disease. Thus, the significance of surgical resection of the primary tumor remains unclear. Since fewer studies have reported on the impact of radiation therapy for the primary lesion in de novo metastatic breast cancer, the benefits of locoregional radiation therapy (LRRT) on the survival are controversial.

The purpose of this article is to summarize the literature, assess the impact of radiation therapy of the primary site on survival in de novo stage IV breast cancer patients, and investigate the prognostic factors in the patients treated with LRRT.

\section{Trends in LRRT for various malignancies with de novo metastatic disease}

Traditionally, radiation therapy has been utilized as palliative treatment for cancer patients with metastatic diseases. However, recent development in not only chemotherapy, surgery, and radiation therapy but also immunotherapy has resulted in a prolongation of the survival time. The clinical benefits of LRRT at the primary site have been reported in patients with metastatic disease at diagnosis in various malignancies.

\section{Prostate cancer}

A British group at Royal Marsden Hospital reported that LRRT improved the median OS for de novo metastatic prostate cancer patients (69 vs. 55.1 months, $\mathrm{P}=0.002$ ) (6). Moreover, the STAMPEDE trial, a randomized controlled phase 3 trial that compared the standard of care for de novo metastatic prostate cancer with or without radiation therapy, showed that radiation therapy improved the failure-free survival $(\mathrm{P}<0.0001)$ but not $\mathrm{OS}(\mathrm{P}=0.266)(7)$.

\section{Non-small cell lung cancer}

Gomez et al. conducted a multicenter randomized trial, which enrolled patients with non-small-cell lung cancer with three or fewer metastases and no progression at 3 or more months after front-line systemic therapy (8). The patients were randomly assigned (1:1) to receive either local consolidative therapy (LCT) or maintenance treatment alone. LCT included LRRT in approximately $80 \%$ of patients. This trial closed early after 49 patients were randomly assigned, due to a significant survival benefit in the local therapy group. They showed a median OS benefit in the LCT arm (median OS: LCT group: 41.2 months vs. no LCT group: 17.0 months, $\mathrm{P}=0.017)$. Moreover, an Italian group published a systematic review and meta-analysis that evaluated the impact on OS when radiation therapy was delivered to the primary lesion in lung cancer patients with oligometastasis (9). The OS and progression free survival (PFS) were improved with the addition of thoracic radiation therapy [OS: hazard ratio (HR) $=0.44, \mathrm{P}<0.001$, PFS: HR $=0.42, \mathrm{P}<0.001]$.

\section{Uterine cervical cancer}

Using the large Surveillance, Epidemiology, and End Results (SEER) database, Huang et al. showed that radiation therapy might provide survival benefits to patients with metastatic uterine cervical cancer (10). Radiation therapy improved the OS $(\mathrm{HR}=0.69)$ and cancer specific survival $(\mathrm{CSS})(\mathrm{HR}=0.79)$ after propensity score matching $(\mathrm{PSM})$ among patients with metastatic cervical cancer. Particularly, the contribution of radiation therapy to survival was significant in patients with one metastatic site.

\section{Rectal cancer}

Liu et al. conducted a retrospective population-based cohort study using the SEER database to evaluate the survival impact of radiation therapy delivered to the primary site in metastatic rectal cancer. The addition of radiation therapy was associated with significantly improved survival benefit after PSM (5-year cause-specific survival: RT group 22\% vs. no RT group $14 \%, \mathrm{P}<0.001)(11)$.

\section{Head and neck cancer}

A Chinese group investigated the role of LRRT in the 
treatment of de novo metastatic nasopharyngeal cancer treated between 1988 and 2012, using the SEER database. At a median follow-up time of 13 months, 448 patients received LRRT, and LRRT was associated with significantly improved OS and CSS in both univariate and multivariate analyses (OS: $\mathrm{HR}=0.50, \mathrm{P}<0.001$; CSS: $\mathrm{HR}=0.50$, $\mathrm{P}<0.001)$, respectively. Moreover, subanalysis showed that younger patients (younger than 65 years), diagnosed after 2003, with non-keratinizing carcinoma or undifferentiated carcinoma [WHO (World Health Organization) type II or III] and who underwent surgery had better survival regarding both OS and CSS (12). A French group also evaluated the benefits of LRRT delivered to the primary tumor in patients with de novo metastatic head and neck squamous cell carcinoma, which consisted of pharyngeal and laryngeal cancers in approximately $80 \%(51 / 64)$ of patients. They showed that the OS was significantly improved for patients who underwent LRRT (median OS 16.1 vs. 7.5 months, $\mathrm{P}<0.01)(13)$.

\section{Reports of LRRT in de novo stage IV breast cancer}

As is the case with other malignant diseases, LRRT for the primary lesion in breast cancer patients with de novo stage IV could be a useful treatment option, but the published data are limited. Since few studies with high levels of evidence have been published so far, the interpretation from retrospective studies will be introduced as follows.

\section{LRT vs. no LRT}

A French group conducted a retrospective study that focused on the impact of LRT on the survival of breast cancer patients with synchronous metastases. Among 581 patients with de novo stage IV disease, 320 received LRT (group A) while 261 received no LRT (group B). LRT consisted of LRRT alone in 249 patients (78\%), surgery of the primary tumor with postoperative LRRT in 41 patients (13\%), and surgery alone in 30 patients (9\%). At a median follow-up time of 39 months, the 3 -year OS rates were $43.4 \%$ and $26.7 \%$ in groups $\mathrm{A}$ and $\mathrm{B}$, respectively $(\mathrm{P}=0.00002)$. LRT was an independent prognostic factor in the multivariate analysis (HR $=0.70,95 \% \mathrm{CI}: 0.58-0.85 ; \mathrm{P}=0.0002)$. The researchers also concluded that LRT was associated with improved survival particularly among patients with visceral metastases, but not among those with bone metastases only (2). Using the SEER database, Ly et al. investigated whether or not LRRT can improve survival among patients with metastatic breast cancer (14). Of 8,761 patients with de novo stage IV disease, radiation therapy was delivered to 1,473 of 3,905 patients who did not undergo surgery, to 882 of 2,070 patients after breast conserving surgery, and to 1,103 of 2,786 patients after mastectomy. The median OS of radiation therapy versus no radiation therapy was 16 vs. 13 months for patients who did not undergo surgery $(\mathrm{P}=0.0003), 28$ vs. 20 months for breast conserving surgery patients $(\mathrm{P}<0.0001)$, and 28 vs. 28 months for mastectomy patients $(\mathrm{P}=0.895)$, respectively. Taken together, the addition of radiation therapy yielded survival benefits among patients without surgery or with breast conserving surgery. Another report by a Canadian group showed the impact of LRT of the primary tumor on survival among patients with de novo stage IV breast cancer (15). The content of LRT was surgery alone in $67 \%$ of patients, radiation therapy alone in $22 \%$, and both in $11 \%$. The 5 -year OS rates were $21 \% / 14 \%(\mathrm{P}<0.001)$ and the 5 -year locoregional PFS rates were $72 \% / 46 \%$ $(\mathrm{P}<0.001)$ among LRT/no LRT patients, respectively. The researchers also showed that among 378 patients treated with LRT, the 5-year OS rates were higher among patients younger than 50 years, with ECOG (Eastern Cooperative Oncology Group) performance status $0-1$, estrogen receptor positive $(\mathrm{ER}+)$ disease, clear surgical margins, single subsite, bone-only metastasis, and one to four metastatic lesions. Choi et al. also assessed the benefits of LRT of the primary lesion in de novo metastatic breast cancer patients (16). LRT consisted of surgery alone in 27 patients, surgery + radiation therapy in 46 patients, and radiation therapy alone in 9 patients, which implies that radiation therapy was used for two-thirds of patients. More favorable outcomes of 5-year OS were obtained in the LRT group compared with the no LRT group $(71 \%$ vs. 40\%, $\mathrm{P}<0.001)$. Moreover, after PSM, the OS of the LRT group remained significantly higher than that of the no LRT group (5-year OS, $73 \%$ vs. $45 \%$; $\mathrm{P}=0.02$ ). Breast conserving surgery + radiation therapy showed more favorable outcomes compared with mastectomy \pm RT [5-year local recurrence-free survival (LRFS), $76 \%$ vs. $62 \%$; $\mathrm{P}=0.05$ ]. However, this could be due to the fact that more patients with favorable prognosis underwent the former treatment. Moreover, a French group evaluated the benefits of LRT on OS in a large retrospective cohort of de novo stage IV breast cancer patients treated between 2008 and 2014. LRT consisted of RT alone ( $\mathrm{n}=518)$, RT + surgery $(\mathrm{n}=523)$, and surgery alone $(n=200)(17)$. LRT was associated with a significantly better OS based on landmark multivariate analysis at 1 -year $(\mathrm{HR}=0.65,95 \% \mathrm{CI}: 0.55-0.76, \mathrm{P}<0.001)$. 
In the subgroup analysis, LRT was correlated with better OS among patients with hormone receptor (HR)-positive/ human epidermal growth factor receptor 2 (HER2)-negative (61.6 vs. 45.9 months, $\mathrm{P}<0.001$ ) and HER2-positive (77.2 vs. 52.6 months, $\mathrm{P}=0.008$ ) tumors, but not among those with triple-negative tumors ( 19 vs. 18.6 months, $\mathrm{P}=0.54$ ). LRT was also associated with a reduction in the risk of death among patients with isolated bone metastasis $(\mathrm{P}<0.001)$ and visceral metastasis without central nervous system involvement $(\mathrm{P}<0.001)$. These results suggested that LRT including radiation therapy would be beneficial in selected breast cancer patients with metastatic lesions at diagnosis. These reports were retrospective studies, and the only one openlabel randomized controlled trial that compared the effect of LRT with no LRT on survival outcome among patients with de novo metastatic breast cancer was conducted in India (4). LRRT was administered to approximately $80 \%$ of patients in the LRT group. LRT resulted in a significant improvement in locoregional PFS compared with that in the no LRT group (median not attained vs. 18.2 months; $\mathrm{P}<0.0001$ ). However, LRT did not show a significant improvement in OS compared with no LRT (median OS: $19.2 v s$. 20.5 months; $\mathrm{P}=0.79$, 2-year OS: $41.9 \%$ vs. $43.0 \%$ ).

\section{Radiation therapy vs. no radiation therapy}

Leung et al. retrospectively assessed whether surgery improves survival among stage IV breast cancer patients. As a subgroup analysis, they also investigated the impact of radiation therapy to the breast on survival. Fifty-eight patients (37\%) received radiation therapy and 99 patients $(63 \%)$ did not, and the median survival duration of both groups were 17 months $(\mathrm{P}=0.21)$, resulting in no survival benefit of radiation therapy of the primary site (18). Another study that evaluated the impact of surgery and LRRT on the prognosis of stage IV breast cancer patients was conducted by a Chinese group (19). Sixtysix patients underwent surgery, while 52 received LRRT. The researchers showed that the utilization of radiation therapy was associated with a significantly better outcome of distant PFS (DFPS) but not a significant improvement in OS (DFPS: $\mathrm{P}=0.034$, OS: $\mathrm{P}=0.18$ ), and the mean $\mathrm{OS}$ of the radiation therapy and no radiation therapy groups were 135.5 and 78.3 months, respectively $(\mathrm{P}=0.19)$.

\section{Radiation therapy alone $v$ s. surgery \pm radiation therapy}

Bourgier et al. evaluated the effect of LRT on local control and survival among breast cancer patients with oligometastatic disease (20). Among 308 de novo stage IV breast cancer patients, 239 were treated with LRT which consisted of LRRT (62\%, n=147, radiation therapy group) and breast and axillary surgery with/without radiation therapy ( $38 \%, n=92$, surgery group). The median followup duration was 6.5 years. Long-standing locoregional response was obtained among $85 \%$ of patients in the radiation therapy group. The 3-year metastasis PFS (MPFS)/OS rates were 20\%/39\% in the radiation therapy group and $39 \% / 57 \%$ in the surgery group, respectively. As the distribution of prognostic factors was quite different between the two groups, a direct comparison of survival was difficult to interpret. Therefore, the researchers adjusted the data according to prognostic factors, and reported no significant differences in MPFS or OS between the two groups. Although it is unclear whether radiation therapy alone or surgery is better as LRT from this study, LRT including radiation therapy alone could provide long-standing locoregional control and could be an important treatment option for selected de novo stage IV patients.

\section{Radiation therapy alone vs. surgery + radiation therapy}

Mauro et al. evaluated the clinical outcomes of patients with stage IV breast cancer treated with local radiation therapy for the primary lesion between 2005 and 2013 (21). More than half $(54 \%)$ of the patients received radiation therapy after breast conserving surgery or mastectomy. Local progression was observed in $22.8 \%$ of the 125 stage IV breast cancer patients during a median follow-up duration of 15 months. The mean OS and local PFS (LoPFS) durations were 23.4 and 45.1 months, respectively. The 3-/5-year OS and LoPFS rates were $21.2 \% / 13.3 \%$ and $67.3 \% / 67.3 \%$, respectively. The 3 -year OS rates among patients who underwent locoregional radiation alone and postoperative radiation therapy were $14.0 \%$ and $31.2 \%(\mathrm{P}=0.047)$. The Karnofsky Performance Status (KPS) $(\mathrm{P}=0.015)$, number of metastatic sites $(\mathrm{P}=0.031)$, hormone therapy $(\mathrm{P}=0.0001)$, and radiation therapy dose $(\mathrm{P}=0.0001)$ were independently correlated with OS. The authors concluded that radiation therapy of the primary site could improve the survival outcome among patients with stage IV disease, particularly those with good KPS, low disease burden (1-3 metastatic lesions), who use hormone therapy, and higher total dose of radiation therapy. 


\section{Surgery + radiation therapy vs. surgery alone}

Kim et al. investigated the survival benefits of postoperative radiation therapy in de novo stage IV breast cancer patients using SEER data base (22). After PSM, the 3-year CSS/OS rates in the no radiation therapy $(\mathrm{n}=882)$ and radiation therapy $(\mathrm{n}=882)$ groups were $57.1 \% / 53.6 \%$ and $70.9 \% / 68.4 \%$, respectively, with better survival outcomes in the radiation therapy group $(\mathrm{P}<0.001)$. On multivariate analysis, postoperative radiation therapy was shown to be a significant prognostic factor $(\mathrm{P}<0.001)$.

\section{Discussion}

Recently, the role of LRRT among selected stage IV breast cancer patients is changing, because OS has greatly improved with the advances in multimodality treatment strategies, and the developments of diagnostic radiology such as MRI and FDG-PET/CT have enabled metastatic lesions, which were difficult to be detected 10-20 years ago, to be identified at initial diagnosis. Moreover, several reports have shown that some breast cancer patients with oligometastases treated with hypofractionated stereotactic body radiation therapy can survive more than 10 years (23). Thus, it is important to properly select de novo stage IV breast cancer patients who could benefit from LRRT. Few high evidence studies such as randomized controlled trials investigating the benefits of LRRT among de novo breast cancer patients have been conducted so far. Several reports showed the favorable impact of LRRT on the survival, but the backgrounds of patients treated with LRRT and no LRRT were quite different and selection bias was very likely. This means that younger patients with better prognostic features are more likely to undergo radiation therapy. The significance of LRRT for de novo stage IV breast cancer patients remains controversial. The survival benefit and prognostic factors of LRRT for de novo stage IV breast cancer patients will be assessed next.

\section{Survival benefit (Figure 1)}

Only one randomized controlled trial evaluating the impact of LRT on the survival of metastatic breast cancer patients has been reported so far. The study concluded that LRT yielded an improvement in locoregional PFS, but did not show a significant improvement in OS compared with no LRT (4). However, the authors made the following points: The OS of both groups was lower than that reported from developed countries. This discrepancy is due to late diagnosis of stage IV breast cancer in India. Moreover, although 107 of 350 patients (31\%) had HER2-positive breast cancer, 98 of those patients (92\%) did not receive anti-HER2 therapy due to financial constraints. Therefore, the treatment used in this clinical trial might be different from that used in developed countries. Thus, the results of this study should be interpreted with caution. Although all the other articles were retrospective, many studies suggested that the LRRT was potentially beneficial for de novo metastatic breast cancer $(2,15-17)$. Two groups evaluated the survival benefit of LRRT on patients' prognoses, but could not show a significant improvement in OS $(18,19)$. However, the patient numbers in both studies were insufficient, and the study conducted by Leung et al. did not clarify the details of the combination of surgery and radiation therapy. The other study showed a significant improvement in distant PFS and OS was not improved significantly, but LRRT tended to improve OS. Another group could not show significant differences between radiation therapy alone or surgery regarding MPFS and OS, but the 3-year OS rates were quite good among 39\% and $57 \%$ of patients in the radiation therapy and surgery groups, respectively. Thus, LRT for breast cancer patients with oligometastatic diseases should be a treatment option (20). A report from Brazil showed that the 3-year OS in the radiation therapy alone group was better than that in the surgery + radiation therapy group. However, the patients with favorable prognoses were categorized into the surgery group, so it is difficult to conclude that surgery before radiation therapy is essential (21). A Korean group reported that postoperative radiation therapy was a significant prognostic factor after PSM using data from the SEER database (22). Many studies have shown that LRRT has the potential to improve the survival of breast cancer patients with de novo stage IV disease. However, most of these articles were from retrospective studies and there might be selection bias in these trials, which means that patients with better PS, lower tumor burden, and younger age should be treated with LRT. Recently, several articles using PSM were published, but even PSM could not exclude selection bias completely.

\section{Prognostic factors (Table 1)}

Several studies have focused on the prognostic factors which influenced the survival of de novo stage IV breast cancer patients treated with LRRT. As regards the 


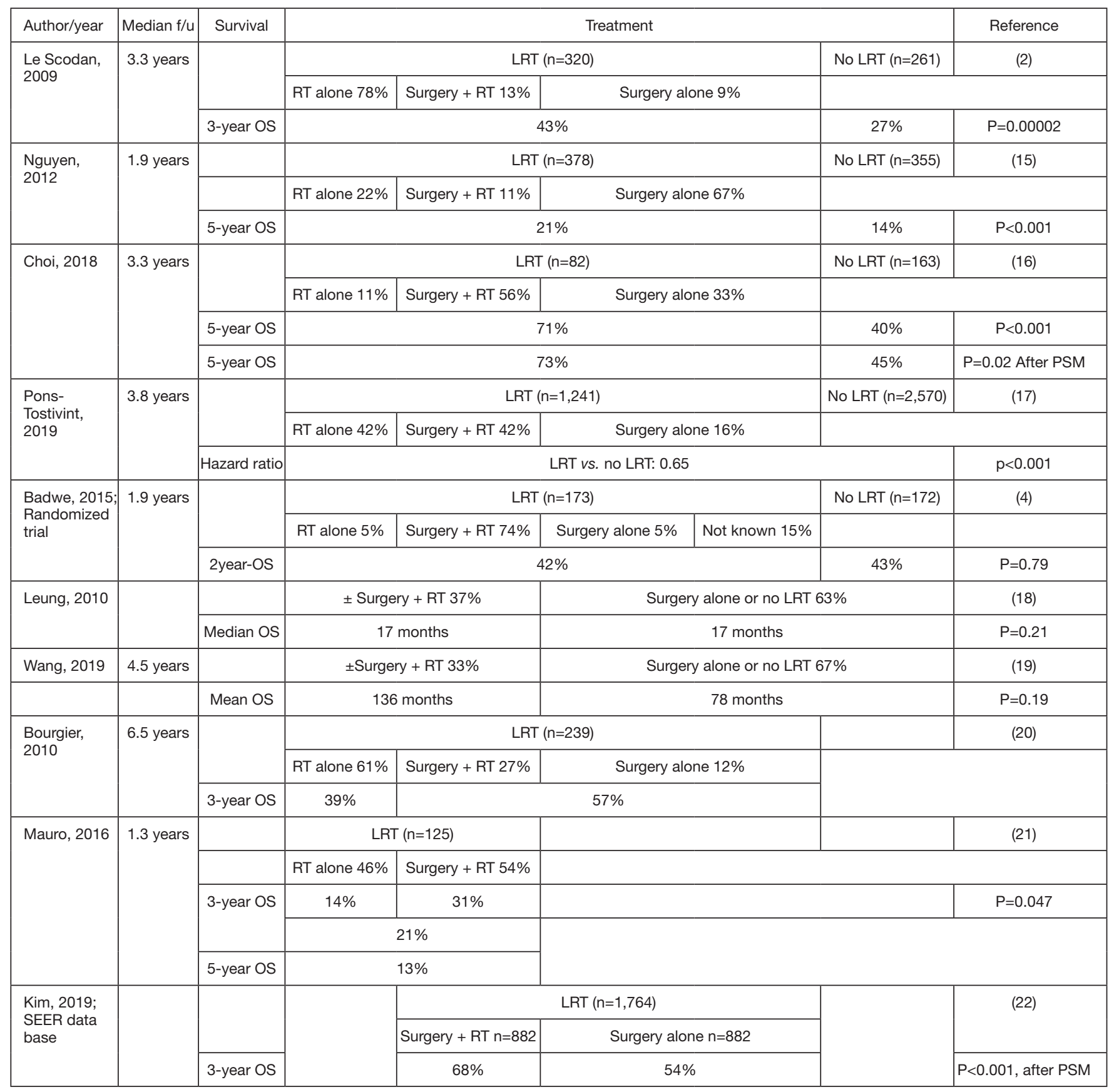

Figure 1 Clinical outcomes of locoregional radiation therapy for patients with de novo stage IV breast cancer (2,4,15-22). OS, overall survival; LRT, locoregional treatment; RT, radiation therapy; LoPFS, local progression free survival; SEER, the Surveillance, Epidemiology, and End Results; PSM, Propensity Score Matching.

metastatic site, the conclusions were conflicting. One group concluded that LRT of the primary lesion could improve the survival, especially in patients with features of poor prognosis with visceral metastases, but not with bone-only metastases (2), while another group showed that LRT was associated with better 5-year OS in patients with boneonly metastasis (15). Another group suggested that isolated bone metastasis and visceral metastasis without central 
Table 1 Favorable Prognostic factors in locoregional radiation therapy for patients with de novo stage IV breast cancer

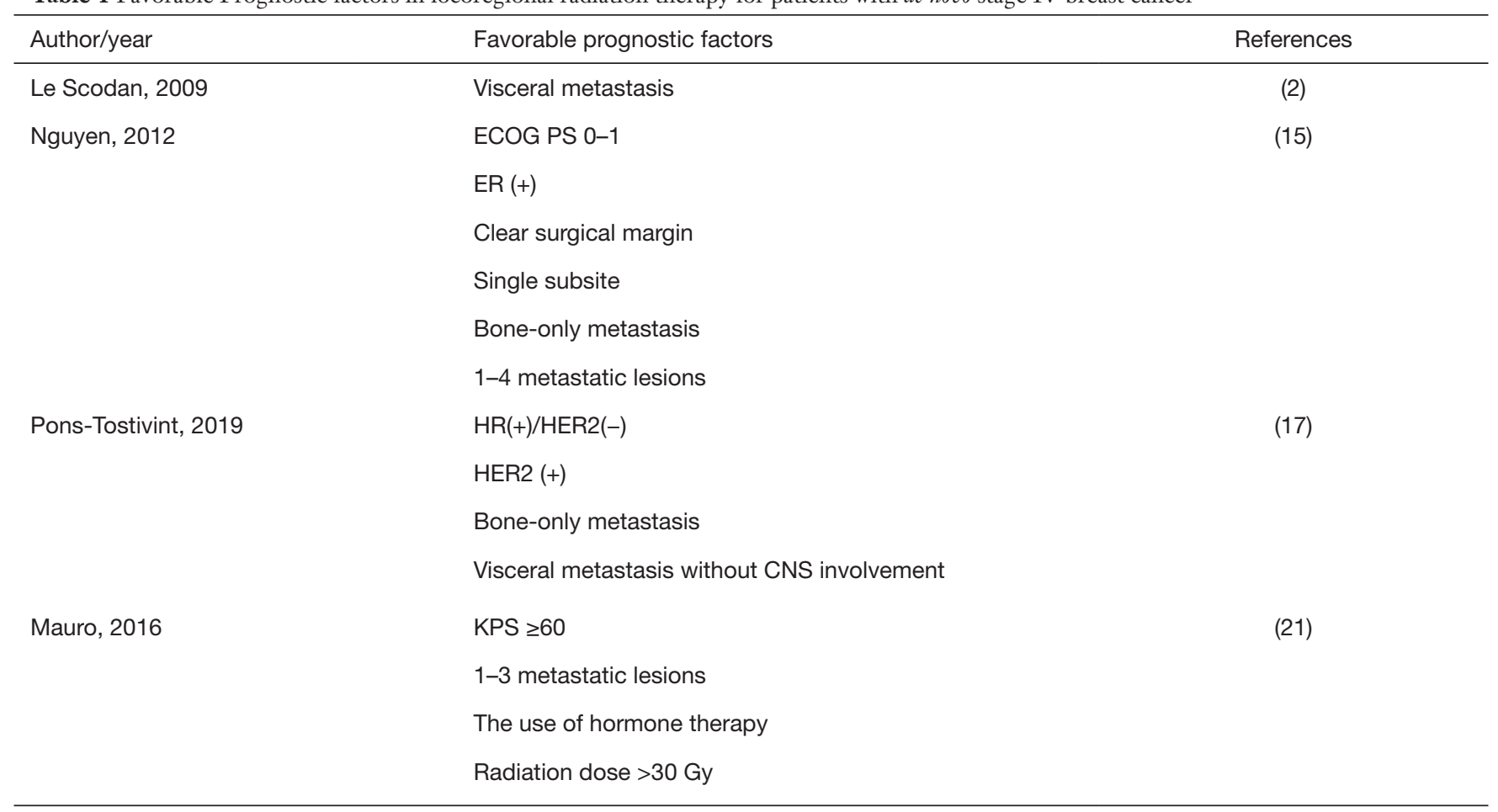

+, positive; -, negative. ECOG PS, Eastern Cooperative Oncology Group Performance Status; ER, estrogen receptor; HR, hormone receptor; HER2, human epidermal growth factor receptor 2.

nervous system involvement was a Favorable prognostic factor (17). Several groups suggested that better performance status is a favorable prognostic factor $(15,17,21)$. Some groups concluded that LRRT could improve the survival of patients with low disease burden $(15,21)$ and younger age was also reported to be a prognostic factor (15). Moreover, several groups investigated the subtype of breast cancer as a prognostic factor. One article pointed out that ER+ was a favorable prognostic factor (15), while the other regarded HR+/HER2- and HER2+, but not triple-negative tumors as having better prognoses (17).

These considerations are almost compatible with those of a recent report (MF07-01) which compared resection of primary tumor with no surgery in stage IV breast cancer at presentation (3). MF07-01 concluded that LRT improved the prognoses of patients with HR(+), HER2(-), younger age ( $<55$ years), and bone-only metastases.

Thus, the prognostic factors for LRRT in de novo stage IV breast cancer patients were controversial. However, all things considered, the selected patients with de novo metastatic disease with better performance status, low tumor burden, younger age, and ER+ could be candidates for LRRT.

\section{Conclusions}

Many retrospective studies suggested that LRRT yields survival benefits among de novo metastatic breast cancer patients. However, since selection bias might be present, it is difficult to interpret the result. Selected de novo stage IV breast cancer patients with better performance status, low tumor burden, younger age, and ER+ disease should be treated with LRRT, but these prognostic factors are not based on absolute evidence because few studies with high evidence levels have been published so far. Only prospective randomized trials can satisfactorily assess the prognostic significance; thus, further randomized trials are eagerly awaited.

\section{Acknowledgments}

Funding: None.

\section{Footnote}

Provenance and Peer Review: This article was commissioned by the Guest Editors (Tadahiko Shien and Kaori Terata) 
for the series "Loco regional therapy for metastatic breast cancer" published in Translational Cancer Research. The article was sent for external peer review organized by the Guest Editors and the editorial office.

Conflicts of Interest: The author has completed the ICMJE uniform disclosure form (available at http://dx.doi. org/10.21037/tcr.2020.02.54).The series "Loco-regional therapy for metastatic breast cancer" was commissioned by the editorial office without any funding or sponsorship. The author has no other conflicts of interest to declare.

Ethical Statement: The author is accountable for all aspects of the work in ensuring that questions related to the accuracy or integrity of any part of the work are appropriately investigated and resolved.

Open Access Statement: This is an Open Access article distributed in accordance with the Creative Commons Attribution-NonCommercial-NoDerivs 4.0 International License (CC BY-NC-ND 4.0), which permits the noncommercial replication and distribution of the article with the strict proviso that no changes or edits are made and the original work is properly cited (including links to both the formal publication through the relevant DOI and the license). See: https://creativecommons.org/licenses/by-nc-nd/4.0/.

\section{References}

1. Thomas A, Khan SA, Chrischilles EA, et al. Initial Surgery and Survival in Stage IV Breast Cancer in the United States, 1988-2011. JAMA Surg 2016;151:424-31.

2. Le Scodan R, Stevens D, Brain E, et al. Breast cancer with synchronous metastases: survival impact of exclusive locoregional radiotherapy. J Clin Oncol 2009;27:1375-81.

3. Soran A, Ozmen V, Ozbas S, et al. Randomized Trial Comparing Resection of Primary Tumor with No Surgery in Stage IV Breast Cancer at Presentation: Protocol MF07-01. Ann Surg Oncol 2018;25:3141-9.

4. Badwe R, Hawaldar R, Nair N, et al. Locoregional treatment versus no treatment of the primary tumour in metastatic breast cancer: an open-label randomised controlled trial. Lancet Oncol 2015;16:1380-8.

5. Fitzal F, Bjelic-Radisic V, Knauer M, et al. Impact of Breast Surgery in Primary Metastasized Breast Cancer: Outcomes of the Prospective Randomized Phase III ABCSG-28 POSYTIVE Trial. Ann Surg 2019;269:1163-9.
6. Bianchini D, Lorente D, Rescigno P, et al. Effect on Overall Survival of Locoregional Treatment in a Cohort of De Novo Metastatic Prostate Cancer Patients: A Single Institution Retrospective Analysis From the Royal Marsden Hospital. Clin Genitourin Cancer 2017;15:e801-e807.

7. Parker CC, James ND, Brawley CD, et al. Radiotherapy to the primary tumour for newly diagnosed, metastatic prostate cancer (STAMPEDE): a randomised controlled phase 3 trial. Lancet 2018;392:2353-66.

8. Gomez DR, Tang C, Zhang J, et al. Local Consolidative Therapy Vs. Maintenance Therapy or Observation for Patients With Oligometastatic Non-SmallCell Lung Cancer: Long-Term Results of a MultiInstitutional, Phase II, Randomized Study. J Clin Oncol 2019;37:1558-65.

9. Petrelli F, Ghidini A, Cabiddu M, et al. Addition of radiotherapy to the primary tumour in oligometastatic NSCLC: A systematic review and meta-analysis. Lung Cancer (Amsterdam, Netherlands) 2018;126:194-200.

10. Huang K, Jia M, Li P, et al. Radiotherapy Improves the Survival of Patients With Metastatic Cervical Cancer: A Propensity-Matched Analysis of SEER Database. Int J Gynecol Cancer 2018;28:1360-8.

11. Liu Q, Shan Z, Luo D, et al. Palliative beam radiotherapy offered real-world survival benefit to metastatic rectal cancer: A large US population-based and propensity scorematched study. J Cancer 2019;10:1216-25.

12. Hu J, Kong L, Gao J, et al. Use of Radiation Therapy in Metastatic Nasopharyngeal Cancer Improves Survival: A SEER Analysis. Sci Rep 2017;7:721.

13. Rambeau A, Bastit V, Thureau S, et al. Impact of locoregional irradiation in patients with upfront metastatic head and neck squamous cell carcinoma. Oral Oncol 2019;93:46-51.

14. Ly BH, Vlastos G, Rapiti E, et al. Local-regional radiotherapy and surgery is associated with a significant survival advantage in metastatic breast cancer patients. Tumori 2010;96:947-54.

15. Nguyen DH, Truong PT, Alexander C, et al. Can locoregional treatment of the primary tumor improve outcomes for women with stage IV breast cancer at diagnosis? Int J Radiat Oncol Biol Phys 2012;84:39-45.

16. Choi SH, Kim JW, Choi J, et al. Locoregional Treatment of the Primary Tumor in Patients With De Novo Stage IV Breast Cancer: A Radiation Oncologist's Perspective. Clin Breast Cancer 2018;18:e167-e178.

17. Pons-Tostivint E, Kirova Y, Lusque A, et al. Survival 
Impact of Locoregional Treatment of the Primary

Tumor in De Novo Metastatic Breast Cancers in a Large

Multicentric Cohort Study: A Propensity Score-Matched Analysis. Ann Surg Oncol 2019;26:356-65.

18. Leung AM, Vu HN, Nguyen KA, et al. Effects of surgical excision on survival of patients with stage IV breast cancer. J Surg Res 2010;161:83-8.

19. Wang W, Liu J, Wang J, et al. Impact of Locoregional Treatment on Prognosis of de novo Stage IV Breast Cancer: A Retrospective Long-Term Study of Chinese Population. Gynecol Obstet Invest 2019;84:248-58.

20. Bourgier C, Khodari W, Vataire AL, et al. Breast radiotherapy as part of loco-regional treatments in stage

Cite this article as: Yoshimura M. Radiation therapy for primary tumor of de novo stage IV breast cancer. Transl Cancer Res 2020;9(8):5108-5116. doi: 10.21037/tcr.2020.02.54
IV breast cancer patients with oligometastatic disease. Radiother Oncol 2010;96:199-203.

21. Mauro GP, de Andrade Carvalho H, Stuart SR, et al. Effects of locoregional radiotherapy in patients with metastatic breast cancer. Breast 2016;28:73-8.

22. Kim YJ, Jung SY, Kim K. Survival benefit of radiotherapy after surgery in de novo stage IV breast cancer: a population-based propensity-score matched analysis. Sci Rep 2019;9:8527.

23. Milano MT, Katz AW, Zhang H, et al. Oligometastatic breast cancer treated with hypofractionated stereotactic radiotherapy: Some patients survive longer than a decade. Radiother Oncol 2019;131:45-51. 